\title{
Conductance calculations with a wavelet basis set
}

\section{Thygesen, Kristian Sommer; Bollinger, Mikkel; Jacobsen, Karsten Wedel}

\section{Published in:}

Physical Review B Condensed Matter

Link to article, DOI:

10.1103/PhysRevB.67.115404

Publication date:

2003

Document Version

Publisher's PDF, also known as Version of record

Link back to DTU Orbit

\section{Citation (APA):}

Thygesen, K. S., Bollinger, M., \& Jacobsen, K. W. (2003). Conductance calculations with a wavelet basis set. Physical Review B Condensed Matter, 67(11), 115404. https://doi.org/10.1103/PhysRevB.67.115404

\section{General rights}

Copyright and moral rights for the publications made accessible in the public portal are retained by the authors and/or other copyright owners and it is a condition of accessing publications that users recognise and abide by the legal requirements associated with these rights.

- Users may download and print one copy of any publication from the public portal for the purpose of private study or research.

- You may not further distribute the material or use it for any profit-making activity or commercial gain

- You may freely distribute the URL identifying the publication in the public portal

If you believe that this document breaches copyright please contact us providing details, and we will remove access to the work immediately and investigate your claim 


\title{
Conductance calculations with a wavelet basis set
}

\author{
K. S. Thygesen, M. V. Bollinger, and K. W. Jacobsen \\ Center for Atomic-scale Materials Physics, Department of Physics, Technical University of Denmark, DK-2800 Kgs. Lyngby, Denmark
}

(Received 30 August 2002; published 12 March 2003)

\begin{abstract}
We present a method based on density functional theory (DFT) for calculating the conductance of a phasecoherent system. The metallic contacts and the central region where the electron scattering occurs, are treated on the same footing taking their full atomic and electronic structure into account. The linear-response conductance is calculated from the Green's function which is represented in terms of a system-independent basis set containing wavelets with compact support. This allows us to rigorously separate the central region from the contacts and to test for convergence in a systematic way. The method supports the use of both norm-conserving and ultrasoft pseudopotentials. We use the method to study the effect of adsorbates on the conductance of an infinitely long, atomically thin $\mathrm{Al}$ wire, and find that hydrogen and oxygen effectively reduce the conductance of the wire by one and two conductance quanta, respectively.
\end{abstract}

DOI: 10.1103/PhysRevB.67.115404

PACS number(s): 73.63. $-\mathrm{b}, 72.15 .-\mathrm{v}, 71.15 .-\mathrm{m}, 85.35 .-\mathrm{p}$

\section{INTRODUCTION}

The study of electron transport in atomic scale systems is becoming increasingly important as the miniaturization of electronic devices proceeds. It is thus of great relevance to establish the connection between the microscopic characteristics of an electronic system, such as the atomic configuration and electronic structure, and transport properties such as the electrical current and conductance. Owing to the complexity of the problem, such studies are strongly dependent on the existence of reliable numerical treatments based on first-principles approaches.

In this paper we present a numerical approach to the calculation of the conductance of a phase-coherent system based on density-functional theory. In the past decade a few different approaches to this problem have been suggested. They all rely on or are equivalent to the Landauer-Büttiker description $^{1}$ of the electronic transport in which the conductance is obtained from the transmission matrix for a scattering region connecting two ballistic leads-and our method is no exception in this respect.

One approach to the scattering problem is to directly calculate the scattering states from which the transmission coefficients can be obtained. This is, for example, the case in the method developed by Lang $^{2}$ where the scattering states for a scattering region connecting two free-electron electrodes are calculated from the Lippman-Schwinger equation. The electronic scattering potential is identified with the effective potential as defined in density-functional theory (DFT), and this potential is then determined self-consistently together with the electron density. The scattering states can alternatively be calculated using a recursion-matrix method as demonstrated by Hirose and Tsukada ${ }^{3}$ or using a wavefunction-matching approach as shown by Choi et al. ${ }^{4,5}$ The scattering-state approach provides a direct real-space picture of the scattering processes, however, only in a few cases a more realistic atomic and electronic structure of the leads has been taken into account. ${ }^{4}$

The conductance can alternatively be calculated within a Green's function approach. The relation between the conductance and the Green's function has for example been derived within the nonequilibrium Keldysh formalism. ${ }^{6}$ This approach requires an explicit separation of the system into leads and a scattering region, and the conductance is then expressed exclusively in terms of the Greens's function for the scattering region. The properties of the leads enter only through self-energy corrections to the Hamiltonian of the scattering region. The approach has been used extensively in connection with tight binding models, where it is straightforward to divide up states as belonging to either leads or scattering region. ${ }^{7-10}$ Recently the Green's function approach has also been combined with DFT with the aim of calculating currents and atomic/electronic structures fully selfconsistently in the presence of an applied bias voltage. The entire system is then described within DFT, using localized basis sets consisting of either atomic orbitals ${ }^{11,12}$ or Gaussians. ${ }^{13}$

In this paper we present a numerical way of calculating the scattering Green's function and the conductance within the DFT framework. The main difference between our method and those of Refs. 11-13 lies in the choice of basis set. While Refs. 11-13 use localized orbital functions centered on the atoms we use a system-independent basis set in the transport direction consisting of wavelets. For the transverse degrees of freedom a basis set consisting of solutions to the two-dimensional Kohn-Sham equation is used. The wavelets have compact support making the separation of leads and scattering region very simple. Furthermore they allow for multiresolution analysis and for a systematic check of basis set convergence.

The method has been implemented in the low-bias linearresponse regime and tested on atomically thin metal wires. In particular we have studied how the adsorption of gas atoms modifies the conductance of an $\mathrm{Al}$ wire. Adsorption of both hydrogen and oxygen is seen to strongly affect the conductance.

The paper is organized as follows. In Sec. II we introduce the Green's function formalism and the expression for the conductance. A general introduction to wavelets including a discussion of the third-order interpolating wavelet used in the present approach is given in Sec. III. We then describe how to generate the basis functions in Sec. IV, and explain in 


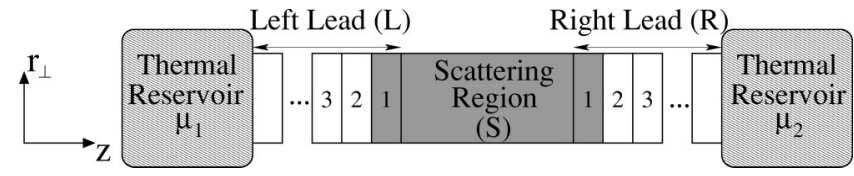

FIG. 1. Schematic setup of the system. It is divided into three regions: the scattering region $(S)$, and the left $(L)$ and right lead $(R)$. The leads connect the scattering region with two thermal reservoirs that are maintained at the chemical potentials $\mu_{1}$ and $\mu_{2}$. The numbering of the leads refers to the division into principal layers.

detail how the connection to DFT is established and in particular implemented in Sec. V. Some tests and applications are presented in Sec. VI, and finally a summary is given in Sec. VII.

\section{GENERAL THEORY}

In this section we describe the theoretical basis for the conductance calculations. The general system setup and the conductance formula are presented, and we discuss how the coupling to the leads is taken into account.

\section{A. System setup and conductance formula}

We consider the transport of non-interacting electrons in a system which can be divided into three regions (see Fig. 1): A scattering region $(S)$, a left lead $(L)$, and a right lead $(R)$. The leads are metallic and connect the scattering region with two thermal reservoirs that are maintained at the chemical potentials $\mu_{1}$ and $\mu_{2}$. The electrons injected from the reservoirs move ballistically through the leads and all scattering events are thus taken to occur in the scattering region. Only elastic scattering is considered and hence the electrons move coherently between the reservoirs. The left and right leads are assumed to couple only indirectly via the scattering region and the Hamiltonian matrix of the entire system therefore takes the form

$$
\boldsymbol{H}=\left(\begin{array}{ccc}
\boldsymbol{H}_{L} & \boldsymbol{H}_{S L}^{\dagger} & 0 \\
\boldsymbol{H}_{S L} & \boldsymbol{H}_{S} & \boldsymbol{H}_{S R} \\
0 & \boldsymbol{H}_{S R}^{\dagger} & \boldsymbol{H}_{R}
\end{array}\right)
$$

where the $\boldsymbol{H}_{i}$ themselves are matrices. The vanishing coupling between the two leads is obtained by using a basis set with compact support in the direction of the electron transport, the $z$ direction in Fig. 1. Furthermore the scattering region has to be large enough so that nonlocal contributions to the Hamiltonian (from, for example, nonlocal pseudopotentials) do not cause a direct coupling.

We shall not assume, however, that the basis functions are orthogonal. The nonorthogonality has been taken explicitly into account in a recent derivation of the conductance formula presented in Ref. 13, where the following definitions and formula are explained in greater detail.

The matrix representation of the retarded Green's function $G^{R}(\varepsilon)$ is defined as ${ }^{14}$

$$
\begin{gathered}
\left(\begin{array}{ccc}
z \boldsymbol{S}_{L}-\boldsymbol{H}_{L} & z \boldsymbol{S}_{S L}^{\dagger}-\boldsymbol{H}_{S L}^{\dagger} & 0 \\
z \boldsymbol{S}_{S L}-\boldsymbol{H}_{S L} & z \boldsymbol{S}_{S}-\boldsymbol{H}_{S} & z \boldsymbol{S}_{S R}-\boldsymbol{H}_{S R} \\
0 & z \boldsymbol{S}_{S R}^{\dagger}-\boldsymbol{H}_{S R}^{\dagger} & z \boldsymbol{S}_{R}-\boldsymbol{H}_{R}
\end{array}\right) \\
\quad \times\left(\begin{array}{ccc}
\boldsymbol{G}_{L}^{R} & \boldsymbol{G}_{L S}^{R} & \boldsymbol{G}_{L R}^{R} \\
\boldsymbol{G}_{S L}^{R} & \boldsymbol{G}_{S}^{R} & \boldsymbol{G}_{S R}^{R} \\
\boldsymbol{G}_{R L}^{R} & \boldsymbol{G}_{R S}^{R} & \boldsymbol{G}_{R}^{R}
\end{array}\right)=\left(\begin{array}{ccc}
\mathbf{1}_{L} & 0 & 0 \\
0 & \mathbf{1}_{S} & 0 \\
0 & 0 & \mathbf{1}_{R}
\end{array}\right),
\end{gathered}
$$

where the $S_{i}$ are overlap matrices and $z=\varepsilon+i \eta, \eta$ being a positive infinitesimal. With this definition the matrix Green's function in general differs from $\left\langle i\left|[(\varepsilon+i \eta) \hat{1}-\hat{H}]^{-1}\right| j\right\rangle$, but the two quantities are related through

$$
\left\langle i\left|[(\varepsilon+i \eta) \hat{1}-\hat{H}]^{-1}\right| j\right\rangle=\left(\boldsymbol{S} \cdot \boldsymbol{G}^{R}(\varepsilon) \boldsymbol{S}\right)_{i j} .
$$

For an orthogonal basis set the matrix Green's function becomes equal to the left hand side of Eq. (3) and the distinction becomes irrelevant.

The current due to noninteracting electrons moving in a (mean-field) electron potential is then given by ${ }^{13}$

$$
\begin{aligned}
I= & \frac{2 e}{h} \int d \varepsilon \operatorname{Tr}\left[\boldsymbol{G}_{S}^{R}(\varepsilon) \boldsymbol{\Gamma}_{L}(\varepsilon) \boldsymbol{G}_{S}^{A}(\varepsilon) \boldsymbol{\Gamma}_{R}(\varepsilon)\right] \\
& \times\left[n_{F}\left(\varepsilon-\mu_{1}\right)-n_{F}\left(\varepsilon-\mu_{2}\right)\right],
\end{aligned}
$$

where $n_{F}(\varepsilon)$ refers to the equilibrium Fermi distribution function and the trace runs over the scattering region only. The central quantity of this relation is the Green's function for the scattering region $G_{S}^{R}(\varepsilon)$

$$
\begin{gathered}
\boldsymbol{G}_{S}^{R}(\varepsilon)=\left[(\varepsilon+i \eta) \boldsymbol{S}_{S}-\boldsymbol{H}_{S}-\boldsymbol{\Sigma}_{L}^{R}(\varepsilon)-\boldsymbol{\Sigma}_{R}^{R}(\varepsilon)\right]^{-1} \\
\boldsymbol{\Sigma}_{i}^{R}(\varepsilon)=\left[(\varepsilon+i \eta) \boldsymbol{S}_{S i}-\boldsymbol{H}_{S i}\right] \boldsymbol{g}_{i}^{(0), R}(\varepsilon) \times\left[(\varepsilon+i \eta) \boldsymbol{S}_{S i}^{\dagger}-\boldsymbol{H}_{S i}^{\dagger}\right] \\
\boldsymbol{g}_{i}^{(0), R}(\varepsilon)=\left[(\varepsilon+i \eta) \boldsymbol{S}_{i}-\boldsymbol{H}_{i}\right]^{-1}, i=(L, R)
\end{gathered}
$$

where $\Sigma_{i}^{R}(\varepsilon)$ is the self energy due to the left ( $\left.i=L\right)$ or right $(i=R)$ lead and $\boldsymbol{g}_{i}^{(0), R}(\varepsilon)$ is the Green's function of the isolated left or right lead. In Eq. (4) the advanced Green's function is given by the usual relation $\boldsymbol{G}_{S}^{A}(\varepsilon)=\left[\boldsymbol{G}_{S}^{R}(\varepsilon)\right]^{\dagger}$ and the $\Gamma_{i}(\varepsilon)$ matrices are obtained from the self-energies

$$
\boldsymbol{\Gamma}_{i}(\varepsilon)=i\left(\boldsymbol{\Sigma}_{i}^{R}(\varepsilon)-\left[\boldsymbol{\Sigma}_{i}^{R}(\varepsilon)\right]^{\dagger}\right) .
$$

The current formula in Eq. (4) is seen to be on the same form as the original expression for the current derived in Ref. 6. The difference is, however, that Eq. (4) is strictly a matrix expression and is derived for the general case where the subspaces of the two leads and the scattering region are allowed to be nonorthogonal.

The relation between the current formula Eq. (4) and the Landauer-Büttiker approach can be established by noting that the transmission matrix $t(\varepsilon)$ is given by ${ }^{15}$

$$
\boldsymbol{t}(\varepsilon)=\left[\boldsymbol{\Gamma}_{R}(\varepsilon)\right]^{1 / 2} \boldsymbol{G}_{S}^{R}(\varepsilon)\left[\boldsymbol{\Gamma}_{L}(\varepsilon)\right]^{1 / 2} .
$$

Substituting this expression into Eq. (9) leads to the well known Landauer-Büttiker formula 


$$
I=\frac{2 e}{h} \int d \varepsilon \operatorname{Tr}\left[\boldsymbol{t}^{\dagger} \boldsymbol{t}\right]\left[n_{F}\left(\varepsilon-\mu_{1}\right)-n_{F}\left(\varepsilon-\mu_{2}\right)\right] .
$$

The current formula in Eq. (4) is also valid for nonequilibrium electron transport. However, this property will not be utilized in the present treatment where we shall focus on the linear response conductance. Assuming the difference between the chemical potentials $\mu_{1}$ and $\mu_{2}$, to be small, the current in Eq. (4) becomes linear in the applied voltage $e U$ $=\left(\mu_{1}-\mu_{2}\right)$. The conductance $G=I / U$ is then given by

$$
G=\frac{2 e^{2}}{h} \operatorname{Tr}\left[\boldsymbol{G}_{S}^{R}(\varepsilon) \boldsymbol{\Gamma}_{L}(\varepsilon) \boldsymbol{G}_{S}^{A}(\varepsilon) \boldsymbol{\Gamma}_{R}(\varepsilon)\right]_{\varepsilon=\varepsilon_{F}},
$$

where the Green's functions are now to be evaluated at the Fermi energy $\varepsilon_{F}$. Equation (9) represents the starting point for the present approach. From Eq. (5) it is seen that in order to obtain the Green's function of the scattering region one needs to calculate the self-energies due to the leads. The calculation of these quantities and in particular the problem of coupling the semi-infinite leads to the scattering region will be addressed in the next section.

\section{B. Coupling to leads}

In the present treatment each of the leads will be taken to be semi-infinite. Since the electrons move ballistically through these regions the leads must be periodic and resemble the bulk metal. As a consequence the scattering region should be chosen sufficiently large so that it comprises all the perturbations on the electron system due to the presence of scatterers. In practice such perturbations are rapidly screened by the electron gas and the mean-field electron potential is expected to decay to its bulk value over a few atomic layers. In the leads the electron potential will have the periodicity of the underlying bulk crystal but in order to calculate the isolated lead Green's function $\boldsymbol{g}_{i}^{(0), R}(\varepsilon)$, one still needs to invert matrices of infinite dimension. This problem is solved by the use of localized basis functions that allows us to divide the leads into so-called principal layers, ${ }^{16,17}$ see Fig. 1. The length of such a layer should be a multiplum of the bulk lattice constant and chosen large enough so that each layer only couples to its two neighboring layers. This also implies that the scattering region will only interact with the first principal layers of the leads, labeled 1 in Fig. 1. The problem of finding $\boldsymbol{g}_{i}^{(0), R}(\varepsilon)$ is thus reduced to that of finding the surface Green's function of the isolated leads, i.e., the Green's function of the first layer. In practice we do this by using the efficient iterative decimation technique. ${ }^{18}$ More details on the calculation of the lead Green's functions are given in Appendix A.

\section{WAVELET THEORY}

This section serves as a brief introduction to the most basic concepts of wavelet theory and multiresolution analysis. The discussion will be limited to the one-dimensional case, however, the theory is readily extended to higher dimensions. We begin by defining the general concept of a multiresolution analysis (MRA). This construction makes precise the idea of analyzing functions on different scales and it offers a natural framework for the discussion of wavelets. After this general introduction, we consider a specific MRA, namely, the one associated with the third-order interpolating wavelet. ${ }^{19}$ This is the wavelet underlying the basis set employed in the present treatment. We end the section with a discussion of some desirable features of this wavelet. A more detailed introduction to wavelets can be found in Ref. 20.

Let $\phi(x)$ be a localized function satisfying

$$
\phi(x)=\sum_{i \in \mathbb{Z}} \alpha_{i} \phi(2 x-i) .
$$

We say that $\phi(x)$ satisfies a two-scale relation with two-scale coefficients $\left\{\alpha_{i}\right\}$. From $\phi(x)$ we can define a sequence of subspaces by

$$
V_{q}=\operatorname{span}\left\{\phi\left(2^{q} x-i\right): i \in \mathbb{Z}\right\} .
$$

The integer $q$ is referred to as the level of the subspace. To characterize these subspaces further, we note that a function $f(x)$ belongs to $V_{0}$ exactly when $f\left(2^{q} x\right)$ belongs to $V_{q}$. Furthermore it follows from Eq. (10) that $V_{q} \subseteq V_{q}$, whenever $q \leqslant q^{\prime}$. The subspaces $\left\{V_{q}\right\}$ provide an increasing sequence of approximation spaces: projecting a function $f$ onto the $q$ th subspace produces an approximation, which converges to $f$ as $q \rightarrow \infty$. The family of subspaces $\left\{V_{q}\right\}$ constitutes an MRA with scaling function $\phi(x)$. If $h$ denotes the width of $\phi$, the approximation space $V_{q}$ will be capable of resolving details down to a length scale of the order $2^{-q} h$.

Suppose $\psi(x)$ is a function in $V_{1}$ such that the subspace $W_{0}=\operatorname{span}\{\psi(x-i): i \in \mathbb{Z}\}$ fulfills the conditions

$$
\begin{aligned}
& V_{0}+W_{0}=V_{1}, \\
& V_{0} \cap W_{0}=\{0\} .
\end{aligned}
$$

Defining the subspaces $\left\{W_{q}\right\}$ according to Eq. (11) with $\phi$ replaced by $\psi$, the following general relations can be shown to hold

$$
\begin{gathered}
V_{q}+W_{q}=V_{q+1}, \\
V_{q} \cap W_{q}=\{0\} .
\end{gathered}
$$

Clearly, the subspace $W_{q}$ contains the details of $V_{q+1}$ not captured by $V_{q}$. For this reason $W_{q}$ is referred to as the detail space at level $q$. By applying these relations repeatedly we obtain the decomposition $\left(q>q_{0}\right)$ :

$$
V_{q}=V_{q_{0}}+W_{q_{0}}+W_{q_{0}+1}+\cdots+W_{q-1},
$$

where all subspaces on the right hand side are mutually disjoint in the sense of Eq. (15). The function $\psi$ is called the wavelet of the MRA. Employing the decomposition Eq. (16), any function $f_{q}$ in $V_{q}$ can be written as a sum of functions each carrying the details of $f_{q}$ at a fixed scale:

$$
f_{q}(x)=f_{q_{0}}(x)+g_{q_{0}}(x)+g_{q_{0}+1}(x)+\cdots+g_{q-1}(x) \text {. }
$$




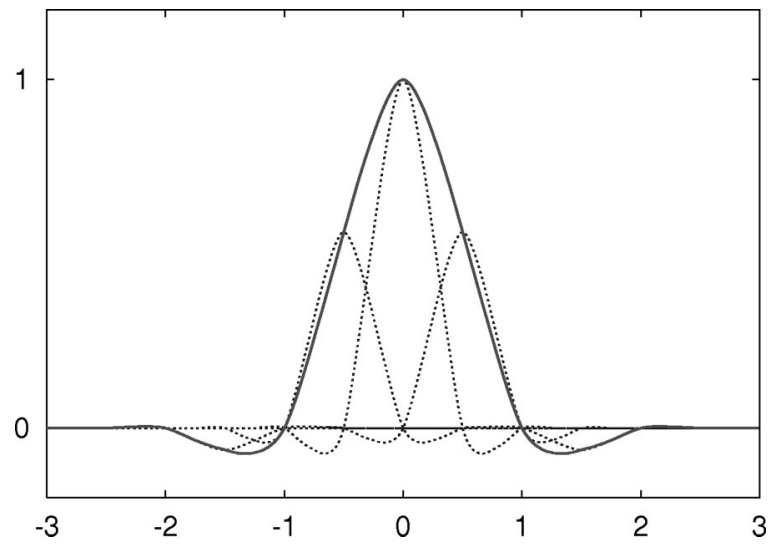

FIG. 2. Illustration of the decomposition expressed by the twoscale relation Eq. (10). The third-order interpolating scaling function (full line) is expanded in terms of its level 1 translates (dotted line).

We have based our numerical scheme on the third-order interpolating scaling function (interpolet) defined and discussed in detail in Refs. 19,21. The relation between this scaling function $\phi$ and its corresponding wavelet $\psi$ is particularly simple: $\psi(x)=\phi(2 x-1)$. Figure 2 shows the form of the interpolet and its decomposition as expressed by the two-scale relation. Apart from fulfilling a two-scale relation, the interpolet has some nice properties: (a) compact support, (b) cardinality, and (c) third-order interpolation. Each of these has useful implications. Property (a) renders the matrix representation of any local operator sparse. Furthermore, it provides a means for decomposing the underlying function space into subspaces containing functions with support in a fixed region of space. This latter point is crucial in our case, since it allows us to separate the scattering region from the leads. Property (b) means that

$$
\phi(i)=\delta_{i, 0}, i \in \mathbb{Z} .
$$

Finally (c) ensures that any third order polynomial, $p(x)$, is exactly reconstructed by an interpolation in terms of $\phi(x-i)$ and its integer translates to

$$
p(x)=\sum_{i \in Z} p(i) \phi(x-i) .
$$

For any function, (b) and (c) together imply that the expansion

$$
\widetilde{f}(x)=\sum_{i \in \mathbb{Z}} f(i) \phi(x-i)
$$

is exact at the integer points, and estimates $f(x)$ to third order at noninteger points. ${ }^{19}$

In the following we consider the problem of obtaining a matrix representation of certain operators which are relevant for applications within quantum mechanics. The representation of a translationally invariant operator $\hat{T}$ with respect to the basis $\{\phi(x-i)\}_{i \in Z}$ is determined by the matrix elements

$$
\boldsymbol{T}_{i, j}=\boldsymbol{T}_{0, j-i}=\int \phi(x) \hat{T} \phi[x-(j-i)] d x .
$$

The numerical values of these matrix elements have been tabulated by $\operatorname{Arias}^{19}$ for operators $\hat{T}=\hat{1}, \hat{T}=d / d x$ and $\hat{T}$ $=d^{2} / d x^{2}$. In the case of a multiplication operator, $\hat{M}_{f}$ the matrix elements take the form

$$
\left(\boldsymbol{M}_{f}\right)_{i, j}=\int \phi(x-i) f(x) \phi(x-j) d x .
$$

The lack of a closed analytic expression for $\phi$, enforces us to introduce an approximation at this point. Thus we invoke the identification

$$
\begin{aligned}
\phi(x-i) f(x) \phi(x-j) \approx & \frac{1}{2} \phi(x-i) f(j) \phi(x-j) \\
& +\frac{1}{2} \phi(x-i) f(i) \phi(x-j),
\end{aligned}
$$

which is clearly a good approximation when $f$ is slowly varying over the support of the interpolet. Within this approximation we obtain for Eq. (22)

$$
\left(\boldsymbol{M}_{f}\right)_{i, j}=\frac{1}{2}[f(i)+f(j)] \boldsymbol{S}_{i, j},
$$

where $S_{i, j}=\langle\phi(x-i), \phi(x-j)\rangle$ is the overlap matrix.

\section{THE BASIS SET}

The general Green's function framework introduced in Sec. II relies on a basic assumption, namely on the compact support of the basis functions in the $z$-direction. In order to facilitate this requirement we introduce the basis functions

$$
|i n\rangle=\phi\left(\frac{z}{d_{z}}-i\right) \chi_{i, n}\left(\boldsymbol{r}_{\perp}\right),
$$

where $\phi$ is the third-order interpolating scaling function and $d_{z}$ is a characteristic length which is roughly given by the scale on which the electron potential varies, see Sec. V. The connection with the theory presented in the preceding section is then established by rescaling the $z$ axis by $d_{z}$. The transverse functions $\chi_{i, n}$ must be linearly independent for fixed $i$, but otherwise the method does not rely on their specific form. Ideally, we would prefer the transverse functions to provide the same resolution as the interpolets do in the transport direction. Depending on the boundary conditions, this could be achieved by two-dimensional interpolets or plane waves. However, using such a basis set the size of the Hamiltonian matrix quickly exceeds the limits of what is computationally tractable. Consequently, we need a way of identifying the most important parts of the transverse subspaces with respect to transport properties.

Inspired by the adiabatic approximation, ${ }^{22,23}$ we have adopted the following procedure. Let $|i \mu\rangle=\phi\left(z / d_{z}\right.$ -i) $\chi_{\mu}\left(\boldsymbol{r}_{\perp}\right)$, where the $\chi_{\mu}$ (not to be confused with $\chi_{i, n}$ ) constitute a complete set of transverse functions. For each $i$ we set up the transverse Hamiltonian and overlap matrices 
$\boldsymbol{H}_{\mu, \mu^{\prime}}^{i}=\left\langle i \mu|\hat{H}| i \mu^{\prime}\right\rangle, \boldsymbol{S}_{\mu, \mu^{\prime}}^{i}=\left\langle i \mu|\hat{S}| i \mu^{\prime}\right\rangle$. In the case of an ultrasoft pseudopotential ${ }^{24}$ the operator $\hat{S}$ includes a nonlocal part due to an augmentation charge, see Sec., but otherwise $\hat{S}=\hat{1}$. Given these matrices we then solve the generalized eigenvalue problem

$$
\boldsymbol{H}^{i} \cdot \mathbf{c}_{n}^{i}=\varepsilon_{n}^{i} \boldsymbol{S}^{i} \cdot \mathbf{c}_{n}^{i}
$$

to obtain the lowest $N_{m}$ transverse eigenstates. The full basis set then consists of the functions of Eq. (25) with the transverse functions at site $i$ given by these eigenstates, i.e.,

$$
\chi_{i, n}\left(\boldsymbol{r}_{\perp}\right)=\sum_{\mu} c_{n, \mu}^{i} \chi_{\mu}\left(\boldsymbol{r}_{\perp}\right), \quad n=1, \ldots, N_{m}
$$

A nice feature of the basis set introduced above, is its ability to capture the exact scattering states for the case of a conductor having a slowly varying potential in the transport direction. For such systems we can apply the adiabatic approximation, which states that a scattering state at energy $E$ takes the form ${ }^{22,23}$

$$
\Psi_{E, n}\left(z, \boldsymbol{r}_{\perp}\right)=\psi_{E, n}(z) \chi_{z, n}\left(\boldsymbol{r}_{\perp}\right),
$$

where $\chi_{z, n}$ for each $z$ is the $n$th eigenfunction of the transverse Hamiltonian $H^{z}=-\frac{1}{2} \partial_{\boldsymbol{r}_{\perp}}^{2}+V\left(z, \boldsymbol{r}_{\perp}\right)$. Within this approximation, the family of transverse modes $\chi_{z, n}$ for a given $n$ constitute a so-called conductance channel. The slow variation of the scattering potential implies that an electron initially injected in a given channel remains in that channel upon traversing the conductor, i.e., no scattering between the channels is induced. Since the conductance is determined by the scattering states at the Fermi level, the ability of the applied basis set to represent these is expected to be an essential issue. In the adiabatic limit we have the following expansion:

$$
\Psi_{E_{F}, n}\left(z, r_{\perp}\right)=\sum_{i} \psi_{E_{F}, n}\left(i d_{z}\right)|i, n\rangle,
$$

which follows from the interpolating property of $\phi$ and the fact that $|i, n\rangle$ is a product of $\phi$ and the $n$th eigenfunction of the transverse Hamiltonian at $z=i d_{z}$.

In the general treatment of realistic atomic scale systems, the scattering between different channels implies that a scattering state can no longer be identified by a single mode, and the number of modes $N_{m}$ which should be included in the basis is not known in advance. It is, however, expected that low-energy modes are more important than high-energy modes. Thus by increasing $N_{m}$ the basis set expands in a well controlled and physically meaningful manner. Moreover, the small overlap between neighboring interpolets and the fact that $\left\langle\chi_{i, n} \mid \chi_{i, m}\right\rangle=\delta_{n, m}$, ensures that the basis set is almost orthogonal, thereby preventing numerical instabilities.

\section{IMPLEMENTATION}

We use the trace formula given in Eq. (9) to calculate the conductance, and in Sec. IV it was argued that $|i n\rangle$ $=\phi\left(z / d_{z}-i\right) \chi_{i, n}\left(\boldsymbol{r}_{\perp}\right)$ is a suitable basis set for evaluating the involved matrices. Consequently the Hamiltonian and overlap matrices entering Eq. (2) take the form $\langle i n|\hat{H}| j m\rangle$ and $\langle i n|\hat{S}| j m\rangle$. As discussed in Sec. I we are treating a system of noninteracting electrons moving in an effective meanfield potential, and the Hamiltonian operator then takes the single-particle form

$$
\hat{H}=-\frac{1}{2} \nabla^{2}+V(\boldsymbol{r})+\hat{V}_{\mathrm{NL}}
$$

where $V(\boldsymbol{r})+\hat{V}_{\mathrm{NL}}$ represents the mean-field potential with $\hat{V}_{\mathrm{NL}}$ denoting a nonlocal pseudopotential.

In the following we present the calculational procedure for obtaining the transverse functions, $\chi_{i, n}\left(\boldsymbol{r}_{\perp}\right)$. We then proceed to describe how the effective DFT potential enters the conductance calculations in practice. Finally a summary is given.

\section{A. Transverse eigenfunctions}

In order to calculate the set of transverse functions $\chi_{i, n}\left(\boldsymbol{r}_{\perp}\right)$, we need for each $i$ to solve the eigenvalue equation given in Eq. (26). With the Hamiltonian of Eq. (30) the eigenvalue problem becomes reminiscent of the twodimensional single-particle Schrödinger equation. We apply the supercell approach ${ }^{25}$ in the transverse plane. The system of interest then constitutes a single cell which is repeated infinitely. In this way a two-dimensional superlattice is formed, and both the potential $V(\boldsymbol{r})$ and the nonlocal pseudopotential operator $\hat{V}_{\mathrm{NL}}$ become periodic with respect to this lattice. The advantage of this approach is that it allows for the modeling of both surfaces, interfaces, and finite systems. In the latter case one just has to make sure that a sufficient amount a vacuum is added between the repeated structures. Using the supercell construction described above we can write the eigenfunctions $\chi_{i, n}\left(\boldsymbol{r}_{\perp}\right)$ as Bloch states

$$
\chi_{i, n, k_{\perp}}\left(\boldsymbol{r}_{\perp}\right)=e^{i k_{\perp} \boldsymbol{r}_{\perp}} u_{n, k_{\perp}}^{i}\left(\boldsymbol{r}_{\perp}\right),
$$

where $u_{n, k_{\perp}}^{i}\left(\boldsymbol{r}_{\perp}\right)$ is a periodic function, $\boldsymbol{k}_{\perp}$ belongs to the two-dimensional first Brillouin zone, and $n$ may be considered as a conductance channel index. Due to the discrete translational symmetry defined by unit vectors of the supercell $\boldsymbol{k}_{\perp}$ is conserved and the Hamiltonian and overlap matrices are diagonal with respect to this variable. This in turn implies that the Green's function is diagonal with respect to $\boldsymbol{k}_{\perp}$, and for a given $\boldsymbol{k}_{\perp}$ we can then independently calculate the conductance $G\left(\boldsymbol{k}_{\perp}\right)$. The total conductance can finally be found by integrating over the two-dimensional Brillouin zone

$$
G=\int \frac{d \boldsymbol{k}_{\perp}}{\Omega_{B Z}} G\left(\boldsymbol{k}_{\perp}\right),
$$

where $\Omega_{B Z}$ is the area of the first Brillouin zone. In practice the integral is carried out by converting it into a finite sum $\int d \boldsymbol{k}_{\perp} / \Omega_{B Z} \rightarrow \Sigma_{\boldsymbol{k}_{\perp}} w\left(\boldsymbol{k}_{\perp}\right)$, where $w\left(\boldsymbol{k}_{\perp}\right)$ represent the weights of the discrete $\boldsymbol{k}_{\perp}$ points. For a given system the applied set of $\boldsymbol{k}_{\perp}$ will depend on both the size and the shape of the 
supercell and their number can often be significantly reduced by symmetry operations, see, e.g., Ref. 26.

The next step is to select a suitable basis set for the evaluation of $u_{n k_{\perp}}^{i}\left(\boldsymbol{r}_{\perp}\right)$. The basis set should span the space of two-dimensional functions having the periodicity of the super lattice. In the current implementation plane waves are used but we stress that this choice is not unique. A different basis set, e.g., two-dimensional, periodic interpolets, could also be applied. Now, expanding the transverse eigenstates in plane waves we obtain

$$
\chi_{i, n, k_{\perp}}\left(\boldsymbol{r}_{\perp}\right)=\sum_{\boldsymbol{G}_{\perp}} c_{n, k_{\perp}}^{i}\left(\boldsymbol{G}_{\perp}\right)\left|\boldsymbol{G}_{\perp}, \boldsymbol{k}_{\perp}\right\rangle,
$$

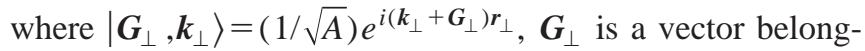
ing to the two-dimensional reciprocal lattice, and $A$ is the area of the supercell. It is noticed that the expansion coefficients $c_{n, k_{\perp}}^{i}\left(\boldsymbol{G}_{\perp}\right)$ in Eq. (33) can be identified with the vector $\mathbf{c}_{n}^{i}$ appearing in Eq. (26). The associated Hamiltonian and overlap matrices are then obtained by replacing $|\mu\rangle$ with $\left|\boldsymbol{G}_{\perp}, \boldsymbol{k}_{\perp}\right\rangle$. In order to make the eigenvalue problem computationally tractable the plane wave expansion needs to be truncated. We do this by including in the plane wave expansion only the $N_{i}$ smallest wave vectors along the direction of each of the two reciprocal lattice vectors $\boldsymbol{G}_{i}$. In this way Eq. (26) has been reduced to an $N_{1} \cdot N_{2}$ dimensional matrix eigenvalue problem and due to its scaling the eigenvectors can be found using standard matrix diagonalization methods. ${ }^{27,28}$

\section{B. Mean-field electron potential}

The mean-field electron potential is obtained from a selfconsistent DFT calculation and this potential then defines the single-particle Hamiltonian of the system. In the present treatment we use the DACAPO pseudopotential $\operatorname{code}^{29}$ to generate the effective potential. It applies a plane wave basis set to iteratively diagonalize the Kohn-Sham equations. More details on the implementation of the code can be found in Refs. 29-32. The DFT calculation is then performed for the scattering region and the first principal layers of the two leads, corresponding to the shaded area in Fig. 1. The supercell approach is applied with periodic boundary conditions in all directions, including the $z$ direction. This means that within the DFT treatment when moving beyond the first principal layer of the right lead one enters the repeated first principal layer of the left lead. If the perturbations originating from the scattering region are properly screened, the system will be periodic in this region and both the electron density and the effective electron potential are thus smoothly joined at the interface between the two leads. This does not exclude the possibility of investigating systems with different leads. In that case one can simply extend the two leads by including additional crystal layers of the respective lead materials in the DFT calculation. The region that now separates the original parts of the leads should be chosen to be sufficiently large so that it comprises all the perturbations due to the presence of the inhomogeneous interface. This spurious part of the electron potential is then simply omitted in the conductance calculation.
Within the conductance calculations the effective DFT potential $V^{\text {eff }}$ is represented on a discrete real space grid. For the plane wave DFT calculation such a representation of the potential is readily obtained using the discrete Fourier transform. The real space resolution of $V^{\text {eff }}$ in turn determines the number and width of the basis functions given in Eq. (25). For the transverse eigenfunctions $\chi_{i, n, k_{\perp}}\left(\boldsymbol{r}_{\perp}\right)$, the discrete real space representation is chosen to have the same form as the transverse part of the effective potential thus the same plane wave expansion is applied to the transverse eigenvalue problem as in the DFT calculation. For the parallel basis functions we initially associate an interpolet with each grid point of $V^{\text {eff }}$ along the $z$-direction. The space spanned by the interpolets localized within the scattering region and the two first principal layer of the leads, i.e., the shaded region in Fig. 1, can be written as

$$
\widetilde{V}_{0}=\operatorname{span}\left\{\phi\left(\frac{z}{d_{z}}-i\right): i \in\left\{0,1, \cdots, N_{z}-1\right\}\right\} \subset V_{0},
$$

where $N_{z}$ is the finite number of discrete real space points along the $z$ direction. $\widetilde{V}_{0}$ is, of course, a subspace of the space $V_{0}$ spanned by the interpolets localized along the $z$ axis of the full system. We let these function spaces represent level 0.

From Eq. (34) it is also seen that the number of scaling functions $N_{z}$ is equal to the number of plane waves used in the DFT calculation along the $z$ direction. For the systems studied so far, it has proved possible to consistently reduce the dimensionality of the wavelet function space. This is achieved by replacing $\widetilde{V}_{0}$ with the coarser $\widetilde{V}_{-1}$ function space

$$
\widetilde{V}_{-1}=\operatorname{span}\left\{\phi\left(\frac{z}{2 d_{z}}-i\right): i \in\left\{0,1, \cdots, \frac{N_{z}}{2}-1\right\}\right\} .
$$

Referring to Eq. (14) it is seen that this replacement corresponds to leaving out the detail space $\widetilde{W}_{-1}$. Even though the interpolets at level -1 are used in the basis set, the effective potential and the projectors associated with the pseudopotential can still be represented using the finer $\widetilde{V}_{0}$ function space along the $z$-axis. This is facilitated by the scaling relation in Eq. (10). More details are given in Appendix B.

\section{Summary}

In the present calculations we apply two versions of the parallel function spaces $\widetilde{V}_{0}$ and $\widetilde{V}_{-1}$ and hence the full threedimensional basis set, Eq. (25) can be rewritten as

$$
\left|i, q, n, \boldsymbol{k}_{\perp}\right\rangle=\phi_{i}^{q}(z) \chi_{i, n, k_{\perp}}\left(\boldsymbol{r}_{\perp}\right), \quad \text { where } \quad(q=-1,0),
$$

where the notation $\phi_{i}^{q}(z)=\phi\left[z /\left(2^{q} d_{z}\right)-i\right]$ has been introduced. Correspondingly the basis set used for solving the transverse eigenvalue equation becomes 


$$
\left|i, q, \boldsymbol{G}_{\perp}, \boldsymbol{k}_{\perp}\right\rangle=\frac{1}{\sqrt{A}} \phi_{i}^{q}(z) e^{i \boldsymbol{G}_{\perp} \cdot \boldsymbol{r}_{\perp}}, \quad \text { where } \quad(q=-1,0) .
$$

For these two basis sets the explicit forms of the inner products associated with the operators $\hat{H}$ and $\hat{S}$ are given in Appendix B. It is noticed that the number $q$ refers to the level of the applied scaling function space. Choosing $q=-1$ reduces the size of the basis set in Eq. (36) by a factor of 2. Consequently the size of the matrices entering Eq. (5) becomes significantly smaller and this in turn allows for the investigation of larger systems that could otherwise not be treated using $q=0$. For a given system consistency between the results obtained for the two function spaces should, of course, always be ensured.

\section{TESTS AND APPLICATIONS}

In this section the derived scheme for calculating the conductance will be applied to a number of different systems. First we investigate a one-dimensional system for which an exact analytical solution exists. We then calculate the conductance of infinite monatomic $\mathrm{Al}$ and $\mathrm{Au}$ wires. These systems serve as test cases for our method. Finally, we investigate how adsorbed atoms affect the conductance of an otherwise perfect $\mathrm{Al}$ wire.

It should be mentioned that for several metals including $\mathrm{Au}$ atomically thin wires can actually be produced. ${ }^{33-35}$ Due to the low coordination of the atoms in such systems, the chemical properties are expected to differ significantly from those of the corresponding bulk systems. In particular the reactivity toward adsorption of light atoms increases, ${ }^{36,37}$ and the presence of impurity atoms must be expected even under ultrahigh vacuum conditions. Under these circumstances conductance measurements might provide information about the actual composition of the wires.

\section{A. One-dimensional system}

We consider a one-dimensional step potential of the form indicated in Fig. 3. For this system the transmission probability for an electron incident with the energy, $\varepsilon$, has an exact analytic expression ${ }^{38}$ and using the Landauer formula the conductance becomes

$$
G(\varepsilon)=\frac{2 e^{2}}{h}\left[1+\frac{V_{0}^{2} \sin ^{2}(\alpha L)}{4 \varepsilon\left(\varepsilon+V_{0}\right)}\right]^{-1},
$$

where $\alpha=\sqrt{2 m\left(\varepsilon+V_{0}\right) / \hbar^{2}}$ and $L, V_{0}$ are the length and depth of the step potential, respectively. In Fig. 3 the calculated values for the conductance are seen to be in complete agreement with the analytic result.

\section{B. Infinite monatomic wires}

We have also investigated the conductance of infinite monatomic wires. Two different metals are used $\mathrm{Al}$ and $\mathrm{Au}$ for which the ionic cores are described by ultrasoft pseudopotentials. ${ }^{24}$ With these psedudopotentials the effec-

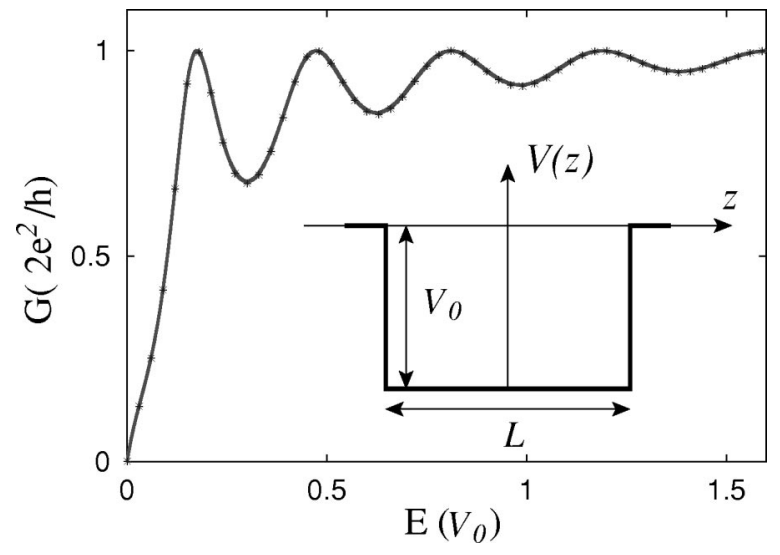

FIG. 3. Conductance of a one-dimensional step potential. The full line represents the exact result while the points indicate the calculated values. The used parameters are $L=16 a_{0}$ and $V_{0}$ $=1.0 \mathrm{Ha}$.

tive Kohn-Sham potentials are obtained from self-consistent DFT calculations for the two systems.

Since the leads are semi-infinite and periodic, the scattering states can in general be identified with left and right moving Bloch states. For the infinite wires the scattering region is identical to a single period in the leads, and therefore the Bloch states do not undergo any scattering at all. According to the Landauer formula the conductance then becomes

$$
G(\varepsilon)=\frac{2 e^{2}}{h} N(\varepsilon),
$$

where $N(\varepsilon)$ denotes the number of bands existing at the energy $\varepsilon$.

The calculated conductance for an inifinite monatomic $\mathrm{Al}$ wire is shown in the upper left panel of Fig. 4. The conduc-
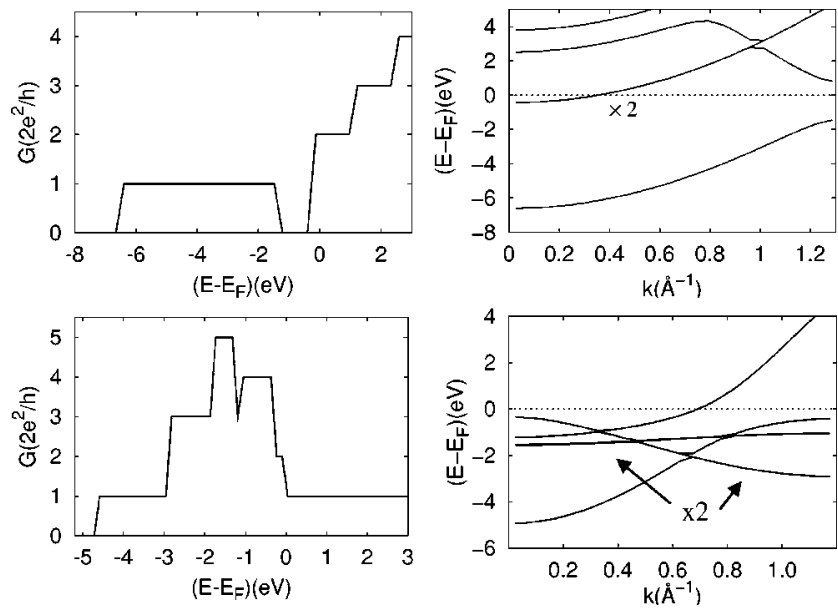

FIG. 4. Infinite monatomic $\mathrm{Al}$ (top) and $\mathrm{Au}$ (bottom) wires. Left: Calculated conductance for a discrete set of energies. Lines have been added as guides to the eye. Right: DFT band structures. The lattice constant of the $\mathrm{Al}(\mathrm{Au})$ wire is $2.39 \AA(2.62 \AA)$ and the repeated wires are separated by $7.5 \AA(6.0 \AA)$. For the conductance calculation $N_{m}=30\left(N_{m}=200\right)$ and the two-dimensional Brillouin zone integration is performed with the $\Gamma$ point only. 


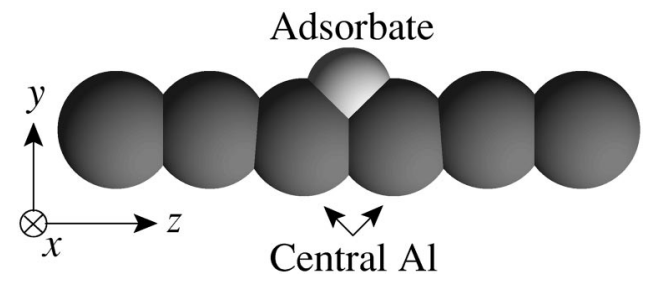

FIG. 5. Al wire with an impurity atom adsorbed on the side. The conductance has been calculated for $\mathrm{H}$ and $\mathrm{O}$ adsorbates.

tance is seen to be quantized in integer steps of $2 e^{2} / h$ as expected from the discussion above. Comparing with the DFT band diagram shown in the upper right part of Fig. 4, these integers are seen to be equal to the number of bands existing in the examined energy window. For future reference, we remark that the states of the doubly degenerate band have $p_{x}$ and $p_{y}$ character, respectively, while the bands just above and below have an $s p_{z}$ character (as usual $z$ denotes the wire axis). The conductance of a monatomic $\mathrm{Au}$ wire has also been investigated and the calculated conductance is shown together with the corresponding DFT band diagram in the lower panel of Fig. 4.

Both conductance calculations have been converged with an error margin of $0.3 \mathrm{eV}$ with respect to the DFT band diagrams. For $\mathrm{Al}$ this was achieved with $N_{m}=30$, whereas Au required $N_{m}=200$. Using a norm-conserving Au pseudopotential does not improve the convergence thus suggesting that the slower convergence is due to the presence of the localized Au $d$ electrons. This, of course, limits the size of systems that can be treated involving transition metal atoms.

\section{Effects of adsorbed impurities}

The presence of adsorbates on a wire causes scattering of the Bloch electrons and consequently the sharp conductance steps displayed by the perfect wire are destroyed. In this section we study this effect in the case of $\mathrm{H}$ and $\mathrm{O}$ adsorbed on an infinite $\mathrm{Al}$ wire.

The configuration is sketched in Fig. 5. The adsorbate and the two nearest $\mathrm{Al}$ atoms were relaxed within a supercell of fixed dimensions. The resulting Kohn-Sham potential was used to model the scattering region in the conductance calculations, while the potential of the perfect $\mathrm{Al}$ wire was used for the leads. In order to ensure charge conservation, the potentials were aligned with respect to the Fermi levels.

Figure 6 shows the calculated conductance for the $\mathrm{Al}$ wire with $\mathrm{H}$ adsorbed. As expected the adsorbate lowers the conductance of the wire. In order to understand the more detailed features of the conductance curve, we have also calculated the atom-projected density of states (ADOS) from the self-consistent DFT eigenstates, see lower panel of Fig. 6. The $s$ orbital of $\mathrm{H}$ has a finite spectral weight coinciding with the $s p_{z}$ band of the wire, thus indicating a hybridization of these states. Much of the $s$ and $p_{z}$ orbitals of the central Al atoms goes into the bound state lying just below the band, and in the conductance curve this is reflected as a reduction of the ideal conductance of about $1 G_{0}$. However, the $\mathrm{Al} s, p_{z}$ orbitals still contribute to the $s p_{z}$ band and thus the current carried by the $s p_{z}$ states is expected to flow through both the

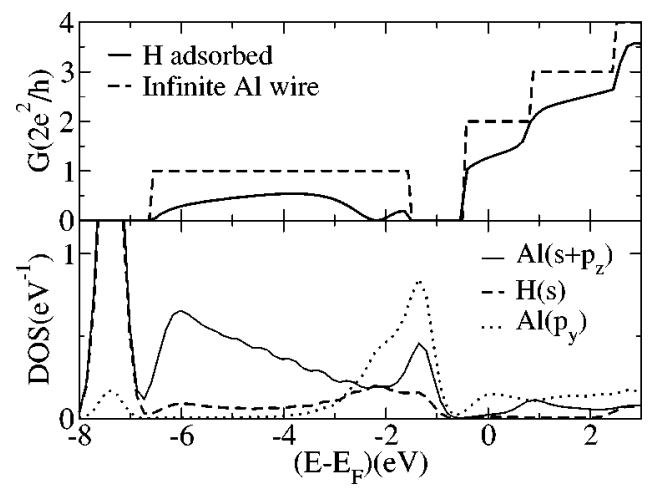

FIG. 6. Conductance of an $\mathrm{Al}$ wire with an $\mathrm{H}$ atom adsorbed on the side. The lower panel shows the DOS projected onto different atomic orbitals of the $\mathrm{H}$ and central $\mathrm{Al}$ atoms.

$\mathrm{H}$ atom and the central $\mathrm{Al}$ atoms. A peculiar feature of the conductance curve is the drop to zero at an energy of $-2.2 \mathrm{eV}$. At this point the projected density of states on the $\mathrm{H} s$ state and the central $\mathrm{Al} s p_{z}$ orbitals are comparable in magnitude and the vanishing conductance is likely to arise because of destructive interference. Above the band gap the hydrogen is seen to cause a lowering of the conductance by approximately $1 G_{0}$, indicating that a channel has been closed. For symmetry reasons, the $p_{x}$ band cannot mix with the other bands, and consequently this channel is expected to remain almost intact. Indeed, the $\mathrm{ADOS}$ for the $\mathrm{Al} p_{x}$ orbitals turn out to differ only slightly from the $p_{x}$ band of the infinite wire. On the other hand the spectral weight of the $p_{y}$ orbitals on the central $\mathrm{Al}$ atoms is shifted down below the bottom of the $p_{y}$ band, thereby effectively closing this channel, see Fig. 6. The calculated conductance with $\mathrm{O}$ adsorbed on the wire is shown in the upper panel of Fig. 7. The lower panel shows the ADOS for the $p$ orbitals of the central Al atoms. The resonance displayed by the conductance at the bottom of the $s p_{z}$ band coincides with peaks in ADOS for the $\mathrm{Al} p_{z}$ orbital and the $\mathrm{O} p_{z}$ orbital (not shown). Above the band gap, the conductance is lowered by approximately $2 G_{0}$, and the ADOS indicate that this is due to a redistribution of the spectral weight of the $p_{x}$ and $p_{y}$ orbitals toward lower energies.

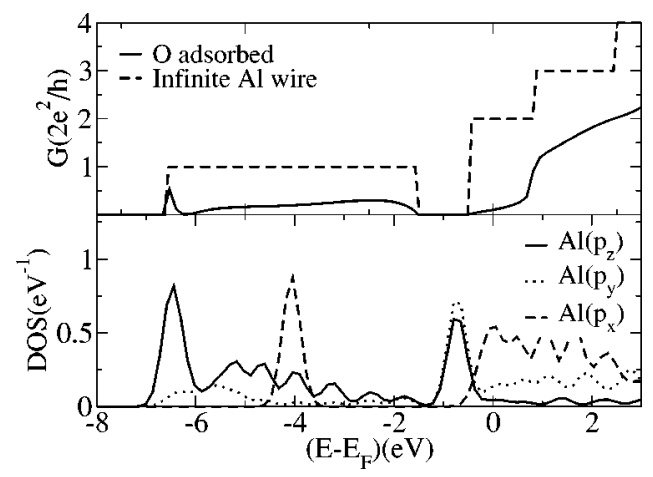

FIG. 7. Conductance of an $\mathrm{Al}$ wire with an $\mathrm{O}$ atom adsorbed on the side. The lower panel shows the DOS projected onto different atomic orbitals of the central $\mathrm{Al}$ atoms. 


\section{SUMMARY}

We have described a numerical method for calculating the conductance of a phase-coherent system in the linearresponse regime. Both the scattering potential and the potential in the ballistic leads are modeled by an effective potential obtained from a self-consistent DFT calculation.

The main focus has been on the numerical implementation. In particular we have given a detailed account for the construction and application of a basis set consisting of wavelets with compact support in the direction of transport and two-dimensional solutions of the Kohn-Sham equation in the transverse plane.

Finally, we have studied the effect of adsorption on the conductance of an infinitely long, atomically thin Al wire, and found that the presence of hydrogen and oxygen effectively reduces the conductance of the wire by, respectively, one and two conductance quanta.

\section{ACKNOWLEDGMENTS}

The Center for Atomic-scale Materials Physics is sponsored by the Danish National Research Foundation. We acknowledge support from the Danish Center for Scientific Computing through Grant No. HDW-1101-05. We thank Lars Bruno Hansen for assistance with the implementation of the ultrasoft pseudopotentials.

\section{APPENDIX A: DETERMINATION OF LEAD GREEN'S FUNCTIONS}

This appendix describes the calculational procedure for finding the isolated lead Green's function $\boldsymbol{g}_{i}^{(0), R}(\varepsilon)$, given in Eq. (5c). In Sec. II B it was argued that the semi-infinte leads can be divided into principal layers that are coupled only to their nearest neighbors. This was facilitated by the use of basis functions with compact support. For the left lead Green's function the following matrix equation is then obtained:

$$
\begin{aligned}
& \left(\begin{array}{cccc}
\ddots & \vdots & \vdots & \vdots \\
\cdots & z \boldsymbol{S}_{3}-\boldsymbol{H}_{3} & z \boldsymbol{S}_{23}^{\dagger}-\boldsymbol{H}_{23}^{\dagger} & 0 \\
\cdots & z \boldsymbol{S}_{23}-\boldsymbol{H}_{23} & z \boldsymbol{S}_{2}-\boldsymbol{H}_{2} & z \boldsymbol{S}_{12}^{\dagger}-\boldsymbol{H}_{12}^{\dagger} \\
\cdots & 0 & z \boldsymbol{S}_{12}-\boldsymbol{H}_{12} & z \boldsymbol{S}_{1}-\boldsymbol{H}_{1}
\end{array}\right) \\
& \times\left(\begin{array}{cccc}
\ddots & \vdots & \vdots & \vdots \\
\cdots & \boldsymbol{g}_{33}^{(0), R} & \boldsymbol{g}_{32}^{(0), R} & \boldsymbol{g}_{31}^{(0), R} \\
\cdots & \boldsymbol{g}_{23}^{(0), R} & \boldsymbol{g}_{22}^{(0), R} & \boldsymbol{g}_{21}^{(0), R} \\
\cdots & \boldsymbol{g}_{13}^{(0), R} & \boldsymbol{g}_{12}^{(0), R} & \boldsymbol{g}_{11}^{(0), R}
\end{array}\right) \\
& =\left(\begin{array}{llll}
\ddots & \vdots & \vdots & \vdots \\
\cdots & \mathbf{1} & 0 & 0 \\
\cdots & 0 & \mathbf{1} & 0 \\
\cdots & 0 & 0 & \mathbf{1}
\end{array}\right)
\end{aligned}
$$

where the subscripts refer to the principal layer in the lead. Due to the periodicity of the electron potential in the leads we have that $\boldsymbol{H}_{i}=\boldsymbol{H}_{1}, \boldsymbol{H}_{i, i+1}=\boldsymbol{H}_{12}$. Similar relations, of course, hold for the overlap matrices. Using the compact notation $\varepsilon^{+}=\varepsilon+i \eta$, the self energy due to the left lead given in Eq. (5b) can be rewritten as

$$
\boldsymbol{\Sigma}_{L}^{R}(\varepsilon)=\left[\varepsilon^{+} \boldsymbol{S}_{S 1}-\boldsymbol{H}_{S 1}\right] \boldsymbol{g}_{11, L}^{(0), R}(\varepsilon)\left[\varepsilon^{+} \boldsymbol{S}_{S 1}^{\dagger}-\boldsymbol{H}_{S 1}^{\dagger}\right]
$$

where the subscript "S1" indicates the matrix elements between the scattering region and the first principle layer. Equation (A2) shows that $\boldsymbol{g}_{11}^{(0), R}$ is the only quantity that needs to be calculated in Eq. (A1). $\boldsymbol{g}_{11}^{(0), R}$ can in turn be obtained from the following set of recursive matrix equations:

$$
\begin{aligned}
{\left[\boldsymbol{\varepsilon}^{+} \boldsymbol{S}_{1}-\boldsymbol{H}_{1}\right] \boldsymbol{g}_{11}^{(0), R}=} & \mathbf{1}-\left[\boldsymbol{\varepsilon}^{+} \boldsymbol{S}_{12}-\boldsymbol{H}_{12}\right] \boldsymbol{g}_{21}^{(0), R} \\
{\left[\varepsilon^{+} \boldsymbol{S}_{1}-\boldsymbol{H}_{1}\right] \boldsymbol{g}_{n 1}^{(0), R}=} & -\left[\varepsilon^{+} \boldsymbol{S}_{12}^{\dagger}-\boldsymbol{H}_{12}^{\dagger}\right] \boldsymbol{g}_{n-1,1}^{(0), R} \\
& -\left[\varepsilon^{+} \boldsymbol{S}_{12}-\boldsymbol{H}_{12}\right] \boldsymbol{g}_{n+1,1}^{(0), R}
\end{aligned}
$$

with $n=2,3, \ldots$. A similar approach applies to the right lead. The iterative decimation technique ${ }^{18}$ provides an efficient and rapidly convergent method for obtaining $\boldsymbol{g}_{11}^{(0), R}$ from the above equations.

\section{APPENDIX B: EVALUATION OF MATRIX ELEMENTS}

In this section the explicit forms of the matrix elements needed for constructing the Hamiltonian and overlap matrices for both the full system, Eq. (2), and for the transverse eigenvalue problem, Eq. (26), are presented. The Hamiltonian operator and the overlap operators have the form, see Sec. V,

$$
\begin{gathered}
\hat{H}=-\frac{1}{2} \nabla^{2}+V(\boldsymbol{r})+\hat{V}_{\mathrm{NL}} \\
\hat{S}=\hat{1}+\hat{Q}_{\mathrm{NL}},
\end{gathered}
$$

where $\hat{V}_{\mathrm{NL}}$ and $\hat{Q}_{\mathrm{NL}}$ are the nonlocal operators associated with the ions described by ultrasoft pseudopotentials. ${ }^{24}$

It is our aim to obtain a matrix representation of these operators with respect to the bases $\left|i, q, n, \boldsymbol{k}_{\perp}\right\rangle$ and $\left|i, q, \boldsymbol{G}_{\perp}, \boldsymbol{k}_{\perp}\right\rangle$, defined in Eqs. (36) and (37). In both cases $q$ represent the level of the applied scaling functions and in the present implementation it can take the values 0 and -1 . By application of the scaling relation (10), the inner products involving $q=-1$ scaling functions can for a general linear operator $\hat{A}$ be rewritten as

$$
\begin{aligned}
& \left\langle i,-1, n, \boldsymbol{k}_{\perp}|\hat{A}| j,-1, m, \boldsymbol{k}_{\perp}\right\rangle \\
& \quad=\sum_{k l} \alpha_{k-i}\left\langle k, 0, n, \boldsymbol{k}_{\perp}|\hat{A}| l, 0, m, \boldsymbol{k}_{\perp}\right\rangle \alpha_{l-j},
\end{aligned}
$$

where the $\alpha_{i}$ are the scaling coefficients. A similar relation holds for the functions $\left|i, q, \boldsymbol{G}_{\perp}, \boldsymbol{k}_{\perp}\right\rangle$. Thus when the $\widetilde{V}_{-1}$ basis set is used, the inner products can be recast as a sum of inner products involving $q=0$ interpolets only. As discussed 
in Sec. V B this allows us to expand, e.g., the local potential in terms of the $\phi_{i}^{0}(z)$ while the interpolets of the basis set belong to the coarser $\widetilde{V}_{-1}$ function space.

In the remainder of this section we state the explicit representations of the operators entering Eq. (B1). We first focus on the local operators $\hat{1}, \nabla^{2}$, and $V(\boldsymbol{r})$ and we then derive the representation for the nonlocal pseudopotential operator. It is noted that the inner products are only given in terms of the $q=0$ basis sets and the $q$ index is thus omitted for clarity. Furthermore, the following notation $\langle\cdot \mid \cdot\rangle_{\perp}=\int d \boldsymbol{r}_{\perp}$ and $\langle$. $|\cdot\rangle_{\|}=\int d z$ is used.

\section{Local operators}

The basis set used for evaluating the full Hamiltonian and overlap matrices yields

$$
\begin{aligned}
\left\langle i, n, \boldsymbol{k}_{\perp}|\hat{1}| j, m, \boldsymbol{k}_{\perp}\right\rangle & =\left\langle\phi_{i} \mid \phi_{j}\right\rangle_{\|},\left\langle\chi_{n, k_{\perp}} \mid \chi_{m, k_{\perp}}\right\rangle_{\perp} \\
\left\langle i, n, \boldsymbol{k}_{\perp}\left|\nabla^{2}\right| j, m, \boldsymbol{k}_{\perp}\right\rangle= & \left\langle\phi_{i}\left|\partial_{z}^{2}\right| \phi_{j}\right\rangle_{\|}\left\langle\chi_{n, k_{\perp}} \mid \chi_{m, k_{\perp}}\right\rangle_{\perp} \\
& +\left\langle\phi_{i} \mid \phi_{j}\right\rangle_{\|}\left\langle\chi_{n, k_{\perp}}\left|\nabla_{\boldsymbol{r}_{\perp}}^{2}\right| \chi_{m, k_{\perp}}\right\rangle_{\perp}, \\
\left\langle i, n, \boldsymbol{k}_{\perp}\left|V\left(z, \boldsymbol{r}_{\perp}\right)\right| j, m, \boldsymbol{k}_{\perp}\right\rangle= & \left\langle\phi_{i} \mid \phi_{j}\right\rangle_{\|} \frac{1}{2}\left\langle\chi_{n, k_{\perp}}\right|\left[V\left(i d_{z}, \boldsymbol{r}_{\perp}\right)\right. \\
& \left.+V\left(j d_{z}, \boldsymbol{r}_{\perp}\right)\right]\left|\chi_{m, k_{\perp}}\right\rangle_{\perp},
\end{aligned}
$$

where in Eq. (B3b) it is used that $\nabla^{2}=\partial_{z}^{2}+\nabla_{\boldsymbol{r}_{\perp}}^{2}$. In order to obtain the expression for the local potential Eq. (24) is applied. For the transverse eigenvalue problem the basis $\left|i, \boldsymbol{G}_{\perp}, \boldsymbol{k}_{\perp}\right\rangle$ is used. The corresponding representations of the local operators now become

$$
\begin{gathered}
\left\langle i, \boldsymbol{G}_{\perp}^{\prime}, \boldsymbol{k}_{\perp}|\hat{1}| i, \boldsymbol{G}_{\perp}, \boldsymbol{k}_{\perp}\right\rangle=\left\langle\boldsymbol{\phi}_{i} \mid \phi_{i}\right\rangle_{\|}\left\langle\boldsymbol{G}_{\perp}^{\prime}, \boldsymbol{k}_{\perp} \mid \boldsymbol{G}_{\perp}, \boldsymbol{k}_{\perp}\right\rangle_{\perp}(\mathrm{B} 4 \mathrm{a}) \\
\left\langle i, \boldsymbol{G}_{\perp}, \boldsymbol{k}_{\perp}\left|\nabla_{\boldsymbol{r}_{\perp}}^{2}\right| i, \boldsymbol{G}_{\perp}^{\prime}, \boldsymbol{k}_{\perp}\right\rangle=\left\langle\phi_{i} \mid \phi_{i}\right\rangle_{\|}\left\langle\boldsymbol{G}_{\perp}^{\prime}, \boldsymbol{k}_{\perp}\left|\nabla_{\boldsymbol{r}_{\perp}}^{2}\right| \boldsymbol{G}_{\perp}, \boldsymbol{k}_{\perp}\right\rangle_{\perp} \\
\left\langle i, \boldsymbol{G}_{\perp}^{\prime}, \boldsymbol{k}_{\perp}\left|V\left(z, \boldsymbol{r}_{\perp}\right)\right| i, \boldsymbol{G}_{\perp}, \boldsymbol{k}_{\perp}\right\rangle \\
=\left\langle\phi_{i} \mid \phi_{i}\right\rangle_{\|}\left\langle\boldsymbol{G}_{\perp}^{\prime}, \boldsymbol{k}_{\perp}\left|V\left(i d_{z}, \boldsymbol{r}_{\perp}\right)\right| \boldsymbol{G}_{\perp}, \boldsymbol{k}_{\perp}\right\rangle_{\perp},
\end{gathered}
$$

where it is noticed in Eq. (B4b) that the parallel part of the Laplacean, $\partial_{z}^{2}$ has been omitted since this term only contributes to the Hamiltonian in the eigenvalue problem with a constant shift in the energy.

\section{Nonlocal operators} is

The general form of the nonlocal pseudopotential operator

$$
\hat{V}_{\mathrm{NL}}=\sum_{l, k, I}\left|\beta_{l}^{I}\right\rangle D_{l, k}^{I}\left\langle\beta_{k}^{I}\right|
$$

where the index $I$ ranges over all ions in the system and $\left|\beta_{l}^{I}\right\rangle$ is a localized function centered at ion $I$. It should be noted that the definition of the non-local operator, $\hat{Q}_{\mathrm{NL}}$ is equivalent to Eq. (B5), the only difference being the matrix elements $D_{l, k}$. Hence we focus here on $\hat{V}_{\mathrm{NL}}$. In the same way as the local electron potential, the projector functions $\beta_{l}^{I}(\boldsymbol{r})$ are evaluated on a real space grid. They can now be expanded as

$$
\beta_{l}(\boldsymbol{r})=\sum_{i, \boldsymbol{G}_{\perp}} \beta_{l}\left(i d_{z}, \boldsymbol{G}_{\perp}\right)\left|i, \boldsymbol{G}_{\perp}\right\rangle,
$$

where $\left|i, \boldsymbol{G}_{\perp}\right\rangle=(1 / \sqrt{A}) \phi_{i}(z) e^{i \boldsymbol{G}_{\perp} \boldsymbol{r}_{\perp}}$. The matrix elements for the nonlocal operator now become

$$
\begin{gathered}
\left\langle i, n, \boldsymbol{k}_{\perp}\left|\hat{V}_{\mathrm{NL}}^{I}\right| j, m, \boldsymbol{k}_{\perp}\right\rangle=\sum_{l, k}\left\langle i, n, \boldsymbol{k}_{\perp} \mid \beta_{l}^{I}\right\rangle D_{l, k}^{I}\left\langle\beta_{k}^{I} \mid j, m, \boldsymbol{k}_{\perp}\right\rangle \\
\left\langle i, \boldsymbol{G}_{\perp}^{\prime}, \boldsymbol{k}_{\perp}\left|\hat{V}_{\mathrm{NL}}^{I}\right| i, \boldsymbol{G}_{\perp}, \boldsymbol{k}_{\perp}\right\rangle \\
=\sum_{l, k}\left\langle i, \boldsymbol{G}_{\perp}^{\prime}, \boldsymbol{k}_{\perp} \mid \beta_{l}^{I}\right\rangle D_{l, k}^{I}\left\langle\beta_{k}^{I} \mid i, \boldsymbol{G}_{\perp}, \boldsymbol{k}_{\perp}\right\rangle,
\end{gathered}
$$

where

$$
\left\langle i, n, \boldsymbol{k}_{\perp} \mid \beta_{l}^{I}\right\rangle=\sum_{j, \boldsymbol{G}_{\perp}^{\prime}}\left\langle\phi_{i} \mid \phi_{j}\right\rangle_{\|}\left\langle\chi_{n, k_{\perp}} \mid \boldsymbol{G}_{\perp}^{\prime}\right\rangle_{\perp} \beta_{l}\left(j d_{z}, \boldsymbol{G}_{\perp}^{\prime}\right)
$$

$$
\left\langle i, \boldsymbol{G}_{\perp}, \boldsymbol{k}_{\perp} \mid \beta_{l}^{I}\right\rangle=\sum_{j, \boldsymbol{G}_{\perp}^{\prime}}\left\langle\phi_{i} \mid \phi_{j}\right\rangle_{\|}\left\langle\boldsymbol{G}_{\perp}, \boldsymbol{k}_{\perp} \mid \boldsymbol{G}_{\perp}^{\prime}\right\rangle_{\perp} \beta_{l}\left(j d_{z}, \boldsymbol{G}_{\perp}^{\prime}\right)
$$

It should be emphasized that when the $\widetilde{V}_{-1}$ function space is used, the projectors just as the local potential can still be expanded in terms of interpolets at level 0 . The matrix elements can then simply obtained by application of Eq. (B2).

\footnotetext{
${ }^{1}$ M. Büttiker, Y. Imry, R. Landauer, and S. Pinhas, Phys. Rev. B 31, 6207 (1985).

${ }^{2}$ N. D. Lang, Phys. Rev. B 52, 5335 (1995).

${ }^{3}$ K. Hirose and M. Tsukada, Phys. Rev. B 51, 5278 (1995).
}

\footnotetext{
${ }^{4}$ H. J. Choi and J. Ihm, Phys. Rev. B 59, 2267 (1999).

${ }^{5}$ Y. G. Yoon, M. S. C. Mazzoni, H. J. Choi, J. Ihm, and S. G. Louie, Phys. Rev. Lett. 86, 688 (2001).

${ }^{6}$ Y. Meir and N. S. Wingreen, Phys. Rev. Lett. 68, 2512 (1992).
} 
${ }^{7}$ T. N. Todorov, G. A. D. Briggs, and A. P. Sutton, J. Phys. C 5, 2389 (1993).

${ }^{8}$ T. N. Todorov, J. Phys.: Condens. Matter 14, 3049 (2002).

${ }^{9}$ J. C. Cuevas, A. L. Yeyati, and A. Martin-Rodero, Phys. Rev. Lett. 80, 1066 (1998).

${ }^{10}$ M. B. Nardelli, Phys. Rev. B 60, 7828 (1999).

${ }^{11}$ M. Brandbyge, J. L. Mozos, P. Ordejón, J. Taylor, and K. Stokbro, Phys. Rev. B 65, 165401 (2002).

${ }^{12}$ J. Taylor, H. Guo, and J. Wang, Phys. Rev. B 63, 245407 (2001).

${ }^{13}$ Y. Xue, S. Datta, and M. A. Ratner, Chem. Phys. 281, 151 (2002).

${ }^{14}$ A. R. Williams, P. J. Feibelman, and N. D. Lang, Phys. Rev. B 26, 5433 (1982).

${ }^{15}$ J. C. Cuevas, A. L. Yeyati, and A. Martín-Rodero, Phys. Rev. Lett. 80, 1066 (1998).

${ }^{16}$ D. H. Lee and J. D. Joannopoulos, Phys. Rev. B 23, 4988 (1981).

${ }^{17}$ D. H. Lee and J. D. Joannopoulos, Phys. Rev. B 23, 4997 (1981).

${ }^{18}$ F. Guinea, C. Tejedor, F. Flores, and E. Louis, Phys. Rev. B 28, 4397 (1983).

${ }^{19}$ T. A. Arias, Rev. Mod. Phys. 71, 267 (1999).

${ }^{20}$ E. Hernandez and G. Weiss, A First Course on Wavelets (CRC, Boca Raton, 1996).

${ }^{21}$ R. A. Lippert, T. A. Arias, and A. Edelman, J. Chem. Phys. 140, 278 (1998).

${ }^{22}$ M. Brandbyge, J. Schiøtz, M. R. Sørensen, P. Stoltze, K. W. Jacobsen, J. K. Nørskov, L. Olesen, E. Laegsgaard, I. Stensgaard, and F. Besenbacher, Phys. Rev. B 52, 8499 (1995).

${ }^{23}$ L. I. Glazman, G. B. Lesovik, D. E. Khmel'nitskii, and R. I. Shekter, JETP Lett. 48, 238 (1988).

${ }^{24}$ D. Vanderbilt, Phys. Rev. B 41, 7892 (1990).
${ }^{25}$ M. C. Payne, M. P. Teter, D. C. Allan, T. A. Arias, and J. D. Joannopoulos, Rev. Mod. Phys. 64, 1045 (1992).

${ }^{26}$ H. J. Monkhorst and J. D. Pack, Phys. Rev. B 13, 5188 (1976).

${ }^{27}$ W. H. Press, S. A. Teukolsky, W. T. Vetterling, and B. P. Flannery, Numerical Recipes in $C$ (Cambridge University Press, Cambridge, 1997).

${ }^{28}$ Linear algebra package(LAPACK), URL http://www.netlib.org/ lapack/.

${ }^{29}$ DACAPO pseudopotential code., URL http://www.fysik.dtu.dk/ campos.

${ }^{30}$ B. Hammer and O. H. Nielsen, in Workshop on Applied Parallel Computing in Physics, Chemistry and Engineering Science (PARA95), edited by J. Wasniewski, Vol. 1041 of Springer Lecture Notes in Computer Science (Springer, Berlin, 1995).

${ }^{31}$ B. Hammer, L. B. Hansen, and J. K. Nørskov, Phys. Rev. B 59, 7413 (1999).

${ }^{32}$ G. Kresse and J. Furthmüller, Comput. Mater. Sci. 6, 15 (1996).

${ }^{33}$ A. I. Yanson, G. Rubio-Bollinger, H. E. van den Brom, N. Agraït, and J. M. van Ruitenbeek, Nature (London) 395, 783 (1998).

${ }^{34}$ H. Ohnishi, Y. Kondo, and K. Takayanagi, Nature (London) 395, 780 (1998).

${ }^{35}$ M. R. Sørensen, M. Brandbyge, and K. W. Jacobsen, Phys. Rev. B 57, 3283 (1998).

${ }^{36}$ S. R. Bahn, N. Lopez, J. K. Nørskov, and K. W. Jacobsen, Phys. Rev. B 66, 081405(R) (2002).

${ }^{37}$ S. B. Legoas, D. S. Galvao, V. Rodrigues, and D. Ugarte, Phys. Rev. Lett. 88, 076105 (2002).

${ }^{38}$ B. H. Bransden and C. J. Joachain, Introduction to Quantum Mechanics (Longman Scientific \& Technical, 1995). 